\title{
Military Real-time Syndromic Surveillance System for Biosurveillance Portal in Korea
}

\author{
ChulWoo Rhee*1, Howard Burkom², Changgyo Yoon', Sangwoo Tak ${ }^{3}$, Aaron Katz ${ }^{2}$ and \\ Miles Stewart ${ }^{2}$
}

${ }^{1}$ Armed Forces Medical Command, Seoul, Korea (the Republic of); ${ }^{2}$ Johns Hopkins Applied Physics Laboratory, Laurel, MD, USA; ${ }^{3}$ Joint Program Executvie Office for Chemical and Biological Defense, Edgewood, MD, USA

\section{Objective}

This presentation aims to elaborate our experiences from initiating a syndromic surveillance system as a part of current biosurveillance developments in Korea. We developed Military Active Realtime Syndromic Surveillance (MARSS) system with data from all of 19 Korean military hospitals as a part of the US-ROK joint Biosurveillance Project.

\section{Introduction}

Biosurveillance Portal (BSP) is a web-based enterprise environment that is aimed to facilitate international collaboration, communication, and information-sharing in support of the detection, management, and mitigation of biological events in Korea. In Oct 2013, Republic of Korea (ROK) Ministry of National Defense has made the project agreement with United States (US) Department of Defense Joint Program Executive Office of Chemical and Biological Defense to develop Biosurveillance Portal which will provide tools and capabilities to facilitate timely identification and detection of biological events to minimize operational impacts on ROK-US Forces. As a part of this project, Armed Forces Medical Command (AFMC) undertook the initiative to develop the Military Active Realtime Syndromic Surveillance system.

\section{Methods}

AFMC currently operates 19 military hospitals across the country, and all electronic health records including patient information, ICD-10 diagnoses and medical prescriptions are collected daily to a centralized server. All the relevant data from Jan 1st 2012 to May 31 st 2014 were retrieved, including total patient counts and counts of patients with specific diagnoses. Seven syndromes were chosen for surveillance based on clinical symptoms and manifestations likely resulting from the suspected use of weaponized pathogens. The selected syndromes are Respiratory, Gastrointestinal, Botulism, Dermatologic, Neurologic, Hemorrhagic, and Fever. The syndrome definitions for diseases associated with critical bioterrorismassociated agents were developed by Center for Disease Control and Prevention (CDC) in Oct, 2003 [1]. All ICD-9 codes were converted to ICD-10 codes based on the General Equivalency Mapping (GEM) files published by Center for Medicare \& Medicaid Services [2].

For development of the syndromic surveillance system, AFMC collaborated with Johns Hopkins University Applied Physics Laboratory (JHUAPL) to modify the Suite for Automated Global Electronic bioSurveillance (SAGES). The underlying statistical methodology of the Electronic Surveillance System for the Early Notification of Community-based Epidemics (ESSENCE) was modified for this purpose. For each syndrome, we performed sensitivity analysis to calculate to obtain practical background alert rates. We estimated positive predictive values (PPV), defined as the proportion of true alert counts during outbreaks to all alert counts, corresponding to these thresholds using effects of known events in the data.

\section{Results}

Corresponding alerting thresholds and other algorithm settings were derived based on the characteristics of MARSS time series using daily counts of ICD-10 codes for each syndrome. For example, series based on the respiratory and fever syndromes clearly showed seasonal variation while series based on the other syndromes did not. Lower algorithm thresholds produced shorter detection delays but also reduced PPV. We chose thresholds with PPV above 30\% to avoid excessive alerting. For the rarer syndromes such as Botulism and Hemorrhagic Illness, true outbreaks are very rare, and algorithm settings were chosen to avoid frequent nuisance alerting.

\section{Conclusions}

Despite the shared surveillance purpose of different syndromic groups, the frequency and seasonality of endemic illness underlying each group should be used for separate algorithm adjustment. For deciding alert thresholds, we have considered not only statistical aspects but also the capacity for alert investigation. More efforts are required to validate the system-generated alerts, and standard operating procedures to investigate these alerts are being developed.

\section{Keywords}

Biosurveillance; Syndromic Surveillance; Bio-threats; SAGES; ESSENCE

\section{Acknowledgments}

This study was funded by Armed Forces Medical Command. (AFMC 2014-024)

\section{References}

1. CDC. Syndrome definitions for diseases associated with critical bioterrorism-associated agents. Atlanta, GA: US Department of Health and Human Services, CDC, 2003. Available at http://www. bt.cdc.gov/surveillance/syndromedef/index.asp.

2. CMS. Official Industry Resources for the ICD-10 Transition. Baltimore, MD: US Department of Health and Human Services, CMS, 2014. Available at http://www.cms.gov/Medicare/Coding/ICD10/index. html.

\section{*ChulWoo Rhee}

E-mail: rhee275@gmail.com 